\title{
Electromyographic assessment of paratonia
}

Lucio Marinelli ${ }^{1}$, Laura Mori ${ }^{1}$, Matteo Pardini ${ }^{1}$, David Beversdorf ${ }^{2}$, Leonardo Cocito ${ }^{1}$, Antonio

Currà $^{3}$, Francesco Fattapposta ${ }^{4}$, Maria Felice Ghilardi ${ }^{5}$, Giovanni Abbruzzese ${ }^{1}$, Carlo Trompetto ${ }^{1}$

${ }^{1}$ Department of Neuroscience, Rehabilitation, Ophthalmology, Genetics, Maternal and Child Health, University of Genova, Largo Daneo 3, 16132 Genova, Italy

${ }^{2}$ University of Missouri Departments of Radiology, Neurology, and Psychological Sciences, and the Thompson Center for Neurodevelopmental Disorders, Columbia, MO, United States of America

${ }^{3}$ Academic Neurology Unit, A. Fiorini Hospital, Terracina (LT), Department of Medical-Surgical

Sciences and Biotechnologies, Sapienza University of Rome, Polo Pontino, Italy

${ }^{4}$ Neurology Unit, Policlinico Umberto I, Department of Neurology and Psichiatry, Sapienza

University of Rome, Rome, Italy

${ }^{5}$ Department of Physiology, Pharmacology and Neuroscience, City University of New York Medical School, New York, NY, United States of America

Corresponding author: Lucio Marinelli, Institute of Neurology, Department of Neuroscience, Rehabilitation, Ophthalmology, Genetics, Maternal and Child Health, University of Genova, Largo Daneo 3, 16132 Genova, Italy. Tel.: +39 010 3537029; Fax: +39 010 3538631; E-mail: lucio.marinelli@unige.it

\section{Acknowledgements}

Dr. Beversdorf is the Thompson Center William and Nancy Thompson Endowed Chair in Radiology

None of the authors have potential conflicts of interest to be disclosed

The article has been published as: Marinelli L, Mori L, Pardini M, Beversdorf D, Cocito L, Currà A, Fattapposta F, Ghilardi MF, Abbruzzese G, Trompetto C. Electromyographic assessment of paratonia. Exp Brain Res. 2017 Mar;235(3):949-956. doi: 10.1007/s00221-016-4854-7. Epub 2016 Dec 20. PubMed PMID: 27999892. 


\begin{abstract}
Many years after its initial description, paratonia remains a poorly understood concept. It is described as the inability to relax muscles during muscle tone assessment with the subject involuntary facilitating or opposing the examiner. Although related to cognitive impairment and frontal lobe function, the underlying mechanisms have not been clarified. Moreover, criteria to distinguish oppositional paratonia from parkinsonian rigidity or spasticity are not yet available. Paratonia is very frequently encountered in clinical practice and only semi-quantitative rating scales are available. The purpose of this study is to assess the feasibility of a quantitative measure of paratonia using surface electromyography. Paratonia was elicited by performing consecutive metronome-synchronized continuous and discontinuous elbow movements in a group of paratonic patients with cognitive impairment. Goniometric and electromyographic recordings were performed on biceps and triceps brachii muscles. Facilitatory (mitgehen) and oppositional (gegenhalten) paratonia could be recorded on both muscles. After normalization with voluntary maximal contraction, biceps showed higher paratonia than triceps. Facilitatory paratonia was higher than oppositional on the biceps. Movement repetition induced increased paratonic burst amplitude only when flexion and extension movements were performed continuously. Both facilitatory and oppositional paratonia increased with movement repetition. Only oppositional paratonia increased following faster movements. This is the first study providing a quantitative and objective characterization of paratonia using electromyography. Unlike parkinsonian rigidity, oppositional paratonia increases with velocity and with consecutive movement repetition. Like spasticity, oppositional paratonia is velocity-dependent, but different from spasticity, it increases during movement repetition instead of decreasing. A quantitative measure of paratonia could help better understanding its pathophysiology and could be used for research purposes on cognitive impairment.
\end{abstract}

Keywords: paratonia; frontal lobe; electromyography; cognitive impairment; spasticity; movements 


\section{Introduction}

Since its first description by E. Dupré in 1910 (Dupré 1910), paratonia has been defined as inability to relax muscles during muscle tone assessment with oppositional paratonia occurring with resistance to passive movements and facilitatory paratonia occurring when the action is in the same direction of passive movement. These two types of paratonia have been originally described separately: Dupré described oppositional paratonia, later called "gegenhalten" (Dupré 1910), while almost 40 years later Kral (Kral 1949) described facilitatory paratonia or "mitgehen" (Mumenthaler 1990). Both types of paratonia have been associated with cognitive impairment or mental disorders (Dupré 1910; Beversdorf and Heilman 1998; Kurlan et al. 2000; Bennett et al. 2002; Damasceno et al. 2005; Vahia et al. 2007). After many years paratonia remains a poorly understood concept. Few studies have been published and the mechanisms underlying paratonia have not yet been clarified. Furthermore, criteria to distinguish oppositional paratonia from parkinsonian rigidity and spasticity are not available, due to its incomplete characterization. Paratonia is very frequently encountered in clinical practice (Hobbelen et al. 2011) but its assessment for research purposes is difficult since only semi-quantitative rating scales are available (Beversdorf and Heilman 1998; Hobbelen et al. 2008). We recently developed a simple neurophysiological approach to study muscle hypertonia, recording EMG responses to manual passive movements performed at different velocities (Marinelli et al. 2013). This method, already validated on patients with spasticity, could be used to differentiate paratonia from other forms of altered muscle tone. Indeed, muscular activity producing facilitation or resistance during passive movements can be recorded using surface electrodes and related to the movement phase by a continuous goniometric recording. The aim of this study is to assess the feasibility of a quantitative and separate measure of facilitatory and oppositional paratonia in a series of patients with clinically assessed paratonia, without parkinsonian rigidity or spasticity.

\section{Materials and methods}

\section{Subjects}

For this proof of concept study, we recruited a convenience sample of ten subjects with cognitive impairment (previously assessed through a complete neuropsychological assessment) and clinically assessed paratonia (score of at least 1 for the paratonia scale or modified Kral procedure, see below) from the out-patient clinic for cognitive disorders at the Department of Neurosciences, University of Genova. Demographic data and clinical characteristics of the enrolled subjects, as well as their clinical diagnosis based on the review of clinical, cognitive and neuroimaging findings are reported in Table 1. Briefly, none of the patients had parkinsonian features, history of stroke or other acute 
brain lesions determined with either a CT or MRI of the brain. As a summary of the cognitive evaluation performed, mini mental status examination (MMSE) and frontal assessment, battery (FAB) values are reported in Table 1 for all subjects. All enrolled subjects underwent a complete neurological examination that included the clinical assessment of facilitatory and oppositional paratonia with a paratonia scale, as well as with the modified Kral procedure (Beversdorf and Heilman 1998). All the patients were able to understand instructions and specifically to remain relaxed during evaluation. All subjects gave informed consent to all study procedures, which were performed according to the declaration of Helsinki. The study was approved by the local ethics committee (San Martino Hospital, Genova, Italy). Patients unable to provide an informed consent were excluded.

\section{Movement evaluation with electromyographic recordings}

The patients were seated in a comfortable armchair with head- and arm-rests so that all patients were completely relaxed during the recordings. The examiner was seated near the patient, was comfortably holding one of the patient's arm, and listened to the tones produced by a softwareemulated metronome through earphones. The examined side was randomly chosen in order to avoid a possible effect of hemispheric dominance. Surface preamplified electrodes with fixed interelectrode distance (TSD150B, Biopac Systems Inc, USA) were placed over the muscle belly of biceps and triceps brachii following current guidelines (Hermens and Roessingh Research and Development BV 1999; Blanc and Dimanico 2010). The distance between the electrodes placed on biceps and triceps muscles was maximized in order to reduce cross-talk. The excursion of elbow joint was continuously recorded by a twin-axis electronic goniometer placed across the joint (TSD130B, Biopac Systems Inc, USA). All signals were acquired by a Biopac MP150 unit (Biopac Systems Inc, USA) for offline analysis.

Continuous and discontinuous elbow movements with a reproducible angular velocity were obtained with a novel method previously described (Marinelli et al. 2013). In the previous validating study involving patients with post-stroke spasticity, we confirmed that this method allows accurate movement timing with a smooth velocity profile and good movement reproducibility over time for a given examiner. During the procedure, subjects were instructed to remain relaxed and to avoid resisting or facilitating the movements performed by the examiner. A baseline recording at rest was performed to confirm the absence of electromyographic activity in both biceps and triceps muscles. Movement velocity and frequency were determined by the metronome (eg. $60 \mathrm{BPM}$ - beats per minute - generating tones at a $1 \mathrm{~s}$ interval). Following the rhythm, the examiner moved the patient's forearm resulting in consecutive, smooth flexionextension of the forearm, reaching the maximal flexion and extension in synchrony with metronome 
tones. The angular velocity of movements was therefore close to that obtained by dividing the joint range of movement (usually $130^{\circ}$ for the elbow) by the metronome tone interval, so that using 60 beats per minute (BPM) the angular velocity was approximately $130 \%$ s. Initially, the examiner performed continuous (or "sinusal") flexion and extension movements in order to adapt the movement velocity to the metronome pacing. Once performance of smooth, continuous movements was perfected, discontinuous (or "linear") movements were obtained by waiting for a few tones before performing the following flexion or extension movement, however preserving accurate timing (Figure 1a). In contrast to the sinusoidal movements, for linear movements it is possible to analyze the electromyographic activity following each movement while the arm is still and without eliciting continuous alternating afferent volleys from muscle spindles of flexor and extensor muscles.

\section{Experimental procedure}

For each patient, we recorded fifteen flexion and extension cycles (i.e. 15 flexions and 15 extensions) for each of the following conditions: 1) sinusoidal movements at 60 BPM, 2) sinusoidal movements at $100 \mathrm{BPM}, 3$ ) linear movements at $60 \mathrm{BPM}, 4)$ linear movements at $100 \mathrm{BPM}$. The four conditions were performed in a random order. We choose 60 and 100 BPM because both are in the range of velocities used for clinical muscle tone evaluation. The use of two different velocities can help to understand if facilitatory and oppositional paratonia are velocity-dependent phenomena. The electromyographic activity resulting from maximal voluntary contraction was also recorded from both biceps and triceps. Each recording condition was preceded by 30 s of resting in order to verify the absence of significant EMG activity before starting passive movements. Considering all patients, a total of 600 flexion and 600 extension movements were therefore analyzed.

\section{Data analysis}

After EMG filtering (notch and band pass 20-250Hz) and rectification, the recordings were first visually inspected offline to exclude the presence of artifacts, to verify the absence of EMG activity before passive movement onsets, and to examine the overall EMG activity between movements when the limb was held steady during linear trials.

EMG amplitude was then measured from the beginning to the end of each movement using the "mean" function of the AcqKnowledge software (version 4.2, Biopac Systems Inc, USA), thus obtaining an average amplitude of bursts independently of movement duration. The maximal voluntary contraction (MVC) of biceps and triceps was also recorded. For each muscle group the subjects were asked to perform 3 maximal contractions lasting $5 \mathrm{~s}$ each and separated by $30 \mathrm{~s}$ rest. Each burst was divided in 500ms time bins and for each bin the mean EMG value was calculated. The highest value among the 3 contractions was considered as the MVC. To allow comparisons 
between muscles and between patients, the average EMG amplitude was normalized to the MVC; the resulting value (mean_EMG) was calculated independently for each muscle and each condition and used for the following analyses.

For each condition, the mean_EMG values were measured and linked to the elbow joint angle so that biceps or triceps bursts appearing during muscle shortening were referred to as facilitatory paratonia, while those appearing during muscle elongation were referred to as oppositional paratonia (Figure 1b).

The mean_EMG values were analyzed using a mixed model ANOVA with condition (linear/sinusoidal), velocity (60/100 BPM), muscle (biceps/triceps) and paratonia type (facilitatory/oppositional) as main factors and movement number (1-15) as within-subjects factor. The correlation between oppositional and facilitatory bursts was assessed using linear regression analysis. Significance level was set at $\mathrm{p}<0.01$. All statistical analyses were performed using StatView version 5.0 (SAS Institute Inc).

\section{Results}

All patients remained relaxed while seated with the examiner at their side holding their arm. There were no complaints of pain or discomfort in the limb; all patients confirmed that, during the testing, they were not actively moving their limb as requested. There were no limitations of the elbow range of motion in any patient, so that the passive movements were performed within an excursion rage of $130^{\circ}$ and their mean velocity was approximately $130 \%$ s at $60 \mathrm{BPM}$ and $217^{\circ} / \mathrm{s}$ at $100 \mathrm{BPM}$.

\section{Facilitatory and oppositional paratonia}

Both facilitatory and oppositional paratonia were visually detectable in the biceps and triceps EMG recordings of all patients in at least one condition. Visual inspection revealed that EMG bursts were rather reproducible within each patient, with a pattern not resembling the triphasic features expected during voluntary movements (Figure 2). Visual inspection of EMG recordings revealed that, during both sinusoidal and linear movements, facilitatory bursts appeared more frequently in the first half of the movement, while oppositional bursts appeared more frequently in the second half (Figure 3a). During sinusoidal movements, with the progressive increase of burst amplitude, oppositional activity near the end of the movement (eg. on the biceps just before reaching complete elbow extension) merged with the facilitatory burst that appears at the beginning of the following movement (eg. biceps activation at the beginning of flexion) (Figure 3b). During linear movements, paratonic bursts usually followed passive movement onset (with a latency of $103 \mathrm{~ms}$ on average), however sometimes EMG bursts occurred simultaneously or even slightly earlier than movement onset. Such early responses are probably intrinsically related to the methodology: at least in some 
occasions, the subject could have predicted the timing of the following movement, despite the examiner randomly interposing a few tone intervals between consecutive movements. Moreover, the examiner could have involuntarily provided a cue just before starting the following movement (eg. slightly increasing grip strength), thus allowing a sensory input preceding the proprioceptive volley related to the actual displacement. The analysis of burst latency should be investigated and discussed in further studies, possibly performed with the aid of robotic devices.

Statistical analysis revealed an overall prominent facilitatory activity, with greatest mean_EMG values for facilitatory paratonia $(\mathrm{F}[1,158]=16.4, \mathrm{p}<0.0001)$. This difference was significant only for the biceps $(F[1,78]=17.1, p<0.0001)$ (Figure 4), while in the triceps both types of paratonia did not differ in amplitude $(\mathrm{F}[1,78]=2.0, \mathrm{p}=0.2)$.

\section{Sinusoidal and linear movements}

Mean_EMG was similar in both sinusoidal and linear movements $(F[1,158]=1.8, p=0.2)$, indicating that both modalities can elicit paratonia. However, we found a significant interaction between condition and movement number $(F[1,14]=6.7, p<0.0001)$, suggesting that the two conditions differ with movement repetition. In fact, unlike linear movements, during the repetition of sinusoidal movements, mean_EMG significantly increased $(F[1,14]=10.3, p<0.0001)$ (Figure 5), a finding also evident in separate analysis for slower $(60 \mathrm{BPM}, \mathrm{F}[39,14]=5.6, \mathrm{p}<0.0001)$ and faster movements (100 BPM, F[39,14]=6.7, $\mathrm{p}<0.0001)$. The progressive increase of mean_EMG during sinusoidal movements was present for both facilitatory $(F[39,14]=6.5, p<0.0001)$ and oppositional $(\mathrm{F}[39,14]=4.1, \mathrm{p}<0.0001)$ bursts and was similar in biceps and triceps muscles $(\mathrm{F}[1,78]=1.2, \mathrm{p}=0.3)$.

\section{The effect of movement velocity}

Faster movements elicited larger bursts (100 BPM versus 60BPM movements: $F[1,158]=5.6$, $\mathrm{p}=0.01$ ), without significant effect of movements repetition (condition $\mathrm{X}$ movement number: $\mathrm{F}[1,14]=1.2, \mathrm{p}=0.3)$. Separate analysis of facilitatory and oppositional activity, however, showed that faster movements elicited significant larger bursts only for oppositional activity $(F[1,78]=6.2$, $\mathrm{p}=0.01)$.

\section{Discussion}

Since the beginning of the previous century, paratonia has been observed during clinical examination and identified as related to cognitive impairment. The distinction between facilitatory and oppositional paratonia was clearly defined only recently, with only facilitatory paratonia found to be related to frontal lobe function (Beversdorf and Heilman 1998). A defective response inhibition, usually associated with lesions of the frontal lobes, was suggested as the main mechanism underlying paratonia. The same study demonstrated that while overall paratonia did not 
correlate with MMSE, specific assessments of facilitatory and oppositional paratonia each did correlate significantly with MMSE (Beversdorf and Heilman 1998), suggesting that the two types of paratonia should be independent measures of a common condition and deserve a more precise and independent assessment.

This is the first study showing that it is possible to obtain a quantitative and objective characterization of paratonia using electromyographic recordings during a testing paradigm. The advantages of this method over existing semi-quantitative scales in terms of unbiased measurement are clear. In fact, the scores of the paratonia scale are based on the examiner's interpretation of definitions like "minimal, moderate, severe and extreme degree of assistance or resistance offered to movement": thus, as in many clinical scales, the examiner's interpretation is a source of increased variability and decreased reproducibility. The modified Kral procedure can provide a more objective assessment only for facilitatory paratonia. Even if rarely considered for the clinical assessment of cognitive impairment, paratonia shows a higher correlation with a number of cognitive tests as compared to primitive reflexes (Beversdorf and Heilman 1998; Damasceno et al. 2005). Moreover, even if a neuropsychological examination is helpful to detect and describe cognitive dysfunction, many tests are influenced by educational factors, motivation and in some cases, malingering (Strauss et al. 2006). These aspects may be less likely to influence the assessment of paratonia, provided the patient's ability to understand the requirement to stay relaxed during the examination.

As stated in the introduction, the main aim of this study was to verify whether a task involving passive elbow movements during EMG recordings and previously validated in spasticity could be used to quantify paratonia in patients with cognitive impairment and clinically detectable paratonia. The EMG recording indeed confirmed the presence of activity referable to paratonia in all patients and allowed us to compare the bursts' amplitude among patients by normalizing the amplitude by the maximal voluntary contraction. In order to distinguish between facilitatory and oppositional components of paratonia, the bursts of biceps and triceps during flexion and extension movements were characterized based on the movement phase and the involved muscle. The highest degree of maximal EMG activity during voluntary contraction was found for the biceps, that indeed has a larger cortical map and lower motor threshold when explored using transcranial magnetic stimulation (Brouwer and Hopkins-Rosseel 1997). After normalization, facilitatory paratonia was higher than oppositional, particularly for the biceps. This finding is in agreement with the idea that facilitatory paratonia is present in the early stages of cognitive impairment, as it was the case in our patient group, while oppositional paratonia appears to be more important in late stages of dementia (Hobbelen et al. 2008; Kleiner-Fisman et al. 2014). The important role of the biceps in producing facilitatory paratonia was suggested by the use of the modified Kral procedure (Beversdorf and 
Heilman 1998), which measures only facilitatory paratonia and involves mostly elbow flexion for the rating.

The evidence emerging from the present study helps to differentiate paratonia from parkinsonian rigidity and spasticity. Parkinsonian rigidity is typically described as velocity-independent and sensitive to dopaminergic treatment (Meara and Cody 1992). Spasticity is defined as a velocitydependent increase in muscle tone due to the exaggeration of the stretch reflex (Lance 1980; Trompetto et al. 2014). Oppositional paratonia appears during passive muscle elongation and can be difficult to distinguish from spasticity and rigidity. We found that oppositional paratonia increases during sinusoidal movements; conversely, spasticity decreases in amplitude during repetitive stretching (AL-Zamil et al. 1995). Nevertheless, in contrast to rigidity, both spasticity and oppositional paratonia are velocity-dependent with increasing amplitude determined by higher velocity. The results of this study support a clearer distinction between oppositional paratonia, rigidity and spasticity.

This exploratory study was conducted in a limited number of subjects with both early cognitive impairment and paratonia to test the feasibility of a neurophysiological approach to study paratonia. Some studies report that paratonia may be detectable also in a minority (about 5\%) of subjects without cognitive impairment (Damasceno et al. 2005; Pauc and Young 2012) using a clinical approach. Like other primitive reflexes, paratonia probably occurs naturally in the newborn and persists in developmental delay and attention deficit disorders instead of disappearing as a consequence of brain development (Pauc and Young 2012). The reappearance of primitive reflexes is probably related to neural loss which may occur with aging and could be eventually related to the development of dementia (Pauc and Young 2012). Paratonia may be an early sign of cognitive impairment and electromyographic assessment could be not only more precise but perhaps could also be more sensitive for its detection compared to a simple clinical approach. In this study we did not include a control group no perform correlations with clinical scales because that was not the purpose of this work; indeed we plan to more comprehensively investigate this issue in future studies following this proof-of-principle. Our results provide elements to better characterize paratonia, encouraging further research to explore the mechanisms underlying this phenomenon.

\section{References}

AL-Zamil ZM, Hassan N, Hassan W (1995) Reduction of Elbow Flexor and Extensor Spasticity Following Muscle Stretch. Neurorehabil Neural Repair 9:161-165. doi: 10.1177/154596839500900305

Bennett HP, Corbett AJ, Gaden S, et al (2002) Subcortical vascular disease and functional decline: a 6-year predictor study. J Am Geriatr Soc 50:1969-1977.

Beversdorf DQ, Heilman KM (1998) Facilitory paratonia and frontal lobe functioning. Neurology 51:968-971. 
Blanc Y, Dimanico U (2010) Electrode Placement in Surface Electromyography (sEMG) ”Minimal Crosstalk Area“ (MCA). Open Rehabil J 3:110-126. doi: 10.2174/1874943701003010110

Brouwer B, Hopkins-Rosseel DH (1997) Motor cortical mapping of proximal upper extremity muscles following spinal cord injury. Spinal Cord 35:205-212.

Damasceno A, Delicio AM, Mazo DFC, et al (2005) Primitive reflexes and cognitive function. Arq Neuropsiquiatr 63:577-582. doi: /S0004-282X2005000400004

Dupré E (1910) Débilité mentale et débilité motrice associées. Rev Neurol (Paris) 20:54-56.

Hermens HJ, Roessingh Research and Development BV (eds) (1999) European recommendations for surface ElectroMyoGraphy: results of the SENIAM project. Roessingh Research and Development, Enschede

Hobbelen JSM, Koopmans RTCM, Verhey FRJ, et al (2008) Diagnosing paratonia in the demented elderly: reliability and validity of the Paratonia Assessment Instrument (PAI). Int Psychogeriatr IPA 20:840-852. doi: $10.1017 / \mathrm{S} 1041610207006424$

Hobbelen JSM, Tan FES, Verhey FRJ, et al (2011) Prevalence, incidence and risk factors of paratonia in patients with dementia: a one-year follow-up study. Int Psychogeriatr IPA 23:1051-1060. doi: $10.1017 / \mathrm{S} 1041610210002449$

Kleiner-Fisman G, Khoo E, Moncrieffe N, et al (2014) A randomized, placebo controlled pilot trial of botulinum toxin for paratonic rigidity in people with advanced cognitive impairment. PloS One 9:e114733. doi: 10.1371/journal.pone.0114733

Kral VA (1949) Ueber eine iterative Bewegunsstörung bei Stirnhirnläsionen. Monatsschrift Für Psychiatr Neurol 118:257-272.

Kurlan R, Richard IH, Papka M, Marshall F (2000) Movement disorders in Alzheimer's disease: more rigidity of definitions is needed. Mov Disord 15:24-29.

Lance JW (1980) Symposium synopsis. In: Spasticity: Disordered Motor Control. Feldman RG, Young RR, Koella WP, pp 485-494

Marinelli L, Trompetto C, Mori L, et al (2013) Manual linear movements to assess spasticity in a clinical setting. PloS One 8:e53627.

Meara RJ, Cody FW (1992) Relationship between electromyographic activity and clinically assessed rigidity studied at the wrist joint in Parkinson's disease. Brain 115 ( Pt 4):1167-1180.

Mumenthaler M (1990) Neurology, 3rd edn. Thieme, Stuttgart

Pauc R, Young A (2012) Paratonia and gegenhalten in childhood and senescence. Clin Chiropr 15:31-34. doi: 10.1016/j.clch.2011.08.001

Strauss E, Sherman EMS, Spreen O, Spreen O (2006) A compendium of neuropsychological tests: administration, norms, and commentary, 3rd ed. Oxford University Press, Oxford; New York

Trompetto C, Marinelli L, Mori L, et al (2014) Pathophysiology of spasticity: implications for neurorehabilitation. BioMed Res Int 2014:354906. doi: 10.1155/2014/354906

Vahia I, Cohen CI, Prehogan A, Memon Z (2007) Prevalence and impact of paratonia in Alzheimer disease in a multiracial sample. Am J Geriatr Psychiatry Off J Am Assoc Geriatr Psychiatry 15:351-353. doi: 10.1097/JGP.0b013e31802ea907

Zehr EP, Sale DG (1994) Ballistic movement: muscle activation and neuromuscular adaptation. Can J Appl Physiol Rev Can Physiol Appliquée 19:363-378. 


\section{Figure legends}

Figure 1

A. Experimental paradigm: metronome tones allowed performing synchronized flexion and extension movements at velocity proportional to metronome BPM value. During the sinusoidal condition, flexion and extension movements were performed continuously without stopping. Instead, during linear movements, a pause of few tones interval was interposed by the examiner between a movement and the following, in order to make the movements discontinuous.

B. Facilitatory (FAC) and oppositional (OPP) activity on biceps and triceps muscle depending on movement direction.

\section{Figure 2}

Sample EMG recordings of facilitatory and oppositional paratonia during continuous (sinusoidal) and discontinuous (linear) movements during a 100 BPM trial. The EMG does not resemble the classical triphasic pattern expected for voluntary movements.

\section{Figure 3}

A. Elbow extension movement during a sample 100 BPM linear condition. The facilitatory burst in the triceps occurs in the first part of the movement, while the oppositional burst in the biceps occurs in the later part.

B. Sample 100 BPM sinusoidal condition. In the biceps, the oppositional burst occurs just before reaching full elbow extension and is immediately followed by a biceps facilitatory burst. During movement repetition, the oppositional burst merges with the subsequent facilitatory burst.

\section{Figure 4}

Biceps mean_EMG average amplitude among the 8 subjects during 15 linear movements performed at 60 BPM. Facilitatory activity (solid line) was higher than oppositional (dashed line) in all movements. The vertical bars represent mean_EMG variability among subjects expressed as standard error.

Figure 5

Mean_EMG amplitude remains stable during linear movements but increases during sinusoidal movements. Sinusoidal (round) and linear (square) trials include all movements (biceps/triceps, 60/100 BPM, facilitatory/oppositional in 10 subjects) and variability is expressed as standard error. 
Table 1 - Clinical features of the recruited patients

\begin{tabular}{|c|c|c|c|c|c|c|c|c|c|c|c|c|}
\hline Patient & Diagnosis & Sex & Age & Edu & Kral_R & Kral_L & PS_f_R & PS_f_L & PS_O_R & PS_O_L & MMSE & FAB \\
\hline 1 & $\mathrm{MCl}$ & $F$ & 82 & 5 & 1 & 1 & 2 & 1 & 2 & 2 & 23 & 9 \\
\hline 2 & $\mathrm{MCl}$ & $M$ & 82 & 5 & 2 & 2 & 2 & 2 & 1 & 1 & 21 & 5 \\
\hline 3 & $\mathrm{MCl}$ & $M$ & 63 & 8 & 0 & 0 & 3 & 3 & 1 & 1 & 27 & 14 \\
\hline 4 & Mixed & $M$ & 80 & 13 & 1 & 1 & 1 & 1 & 1 & 1 & 16 & 8 \\
\hline 5 & $\mathrm{MCl}$ & $M$ & 75 & 13 & 1 & 1 & 1 & 1 & 0 & 1 & 26 & 15 \\
\hline 6 & $A D$ & $M$ & 78 & 5 & 4 & 4 & 3 & 3 & 2 & 3 & 19 & 9 \\
\hline 7 & $\mathrm{MCl}$ & $F$ & 70 & 7 & 0 & 0 & 2 & 2 & 0 & 0 & 21 & 12 \\
\hline 8 & FLD & $M$ & 67 & 8 & 0 & 1 & 3 & 2 & 0 & 0 & 27 & 14 \\
\hline 9 & $\mathrm{MCl}$ & $F$ & 84 & 7 & 1 & 1 & 3 & 3 & 0 & 0 & 26 & 13 \\
\hline 10 & $\mathrm{MCl}$ & $F$ & 74 & 8 & 2 & 2 & 3 & 3 & 1 & 1 & 22 & 9 \\
\hline
\end{tabular}

MCI: mild cognitive impairment; Mixed: mixed vascular and degenerative dementia; AD: Alzheimer's disease; FLD: frontal lobe dementia (mild). Edu: education (years of school); Kral: modified Kral procedure score (left/right arm; 0-4); PS_f: paratonia scale for facilitatory paratonia (left/right arm; 0-4); PS_o: paratonia scale for oppositional paratonia (left/right arm; 0-4). MMSE: mini mental status examination (0-30); FAB: frontal assessment battery (0-18). 
A

FIGURE 1

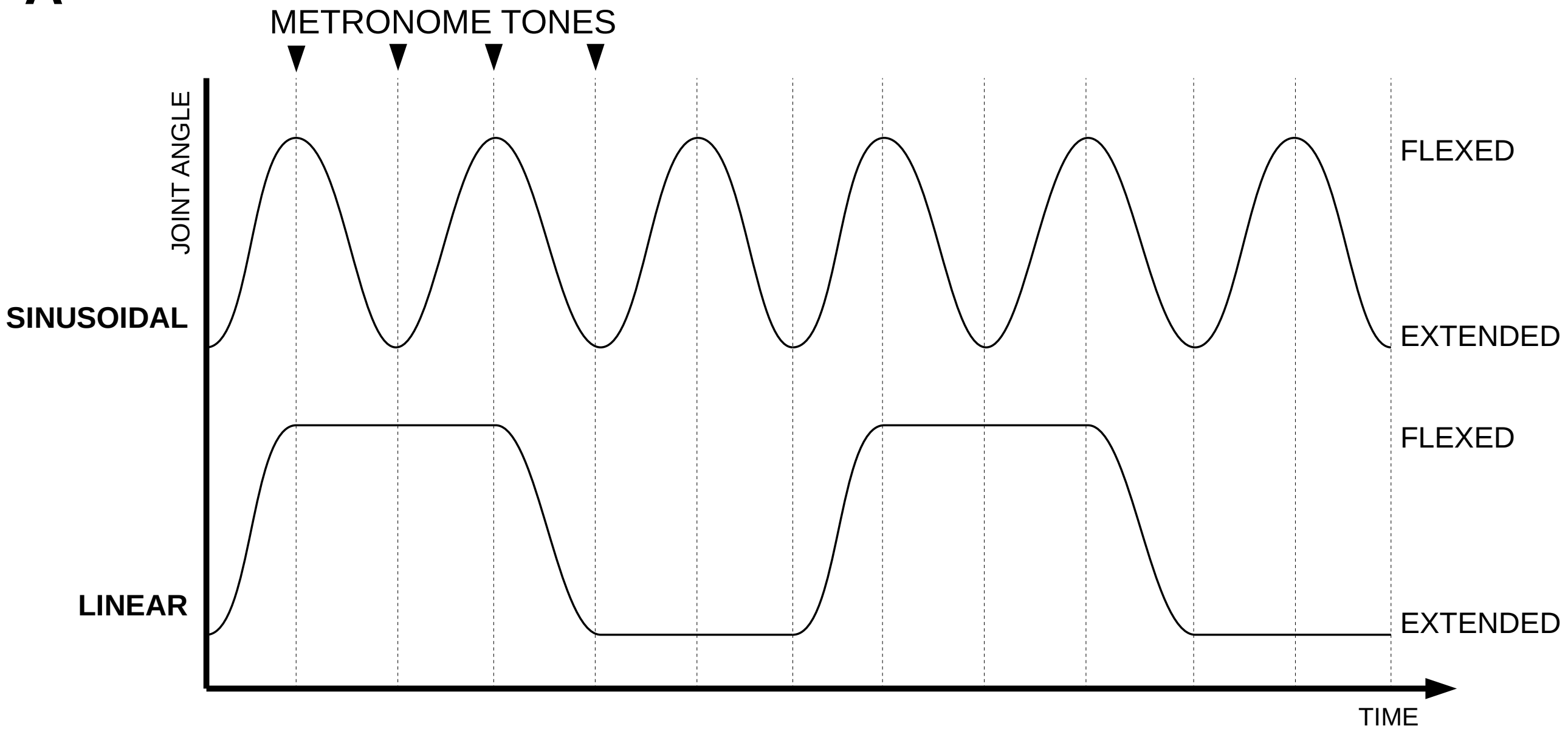

B

\begin{tabular}{|c|c|c|}
\hline & \multicolumn{2}{|c|}{ FLEXED POSITION } \\
\hline BICEPS & FACILITATORY & OPPOSITIONAL \\
\hline TRICEPS & OPPOSITIONAL & FACILITATORY \\
\hline
\end{tabular}


FIGURE 2

SINUSOIDAL

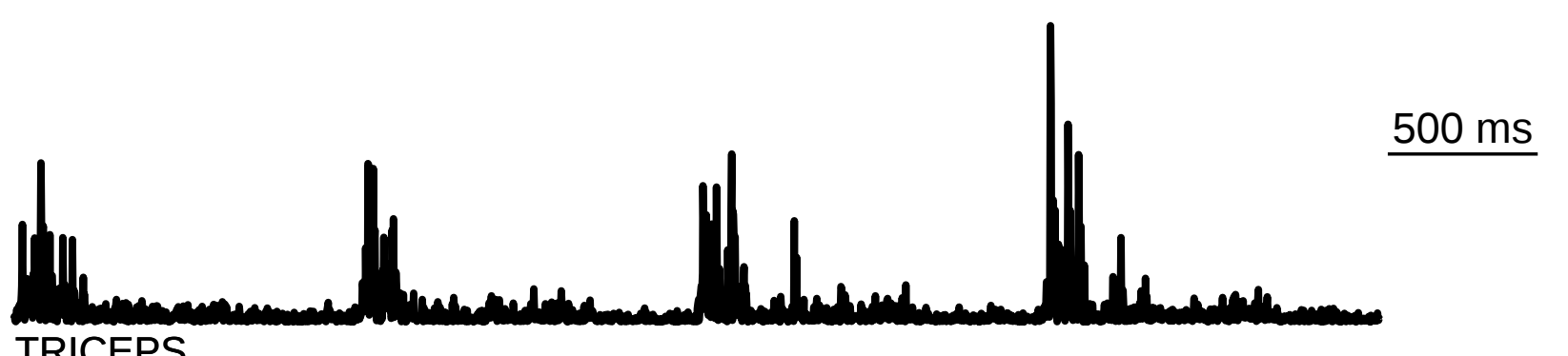
TRICEPS

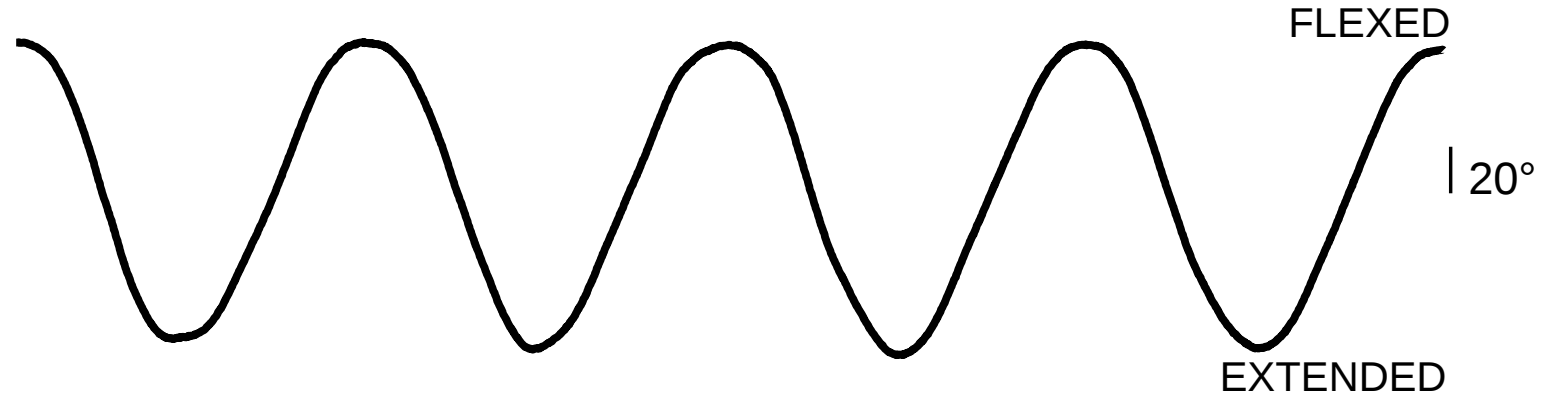

\section{LINEAR}
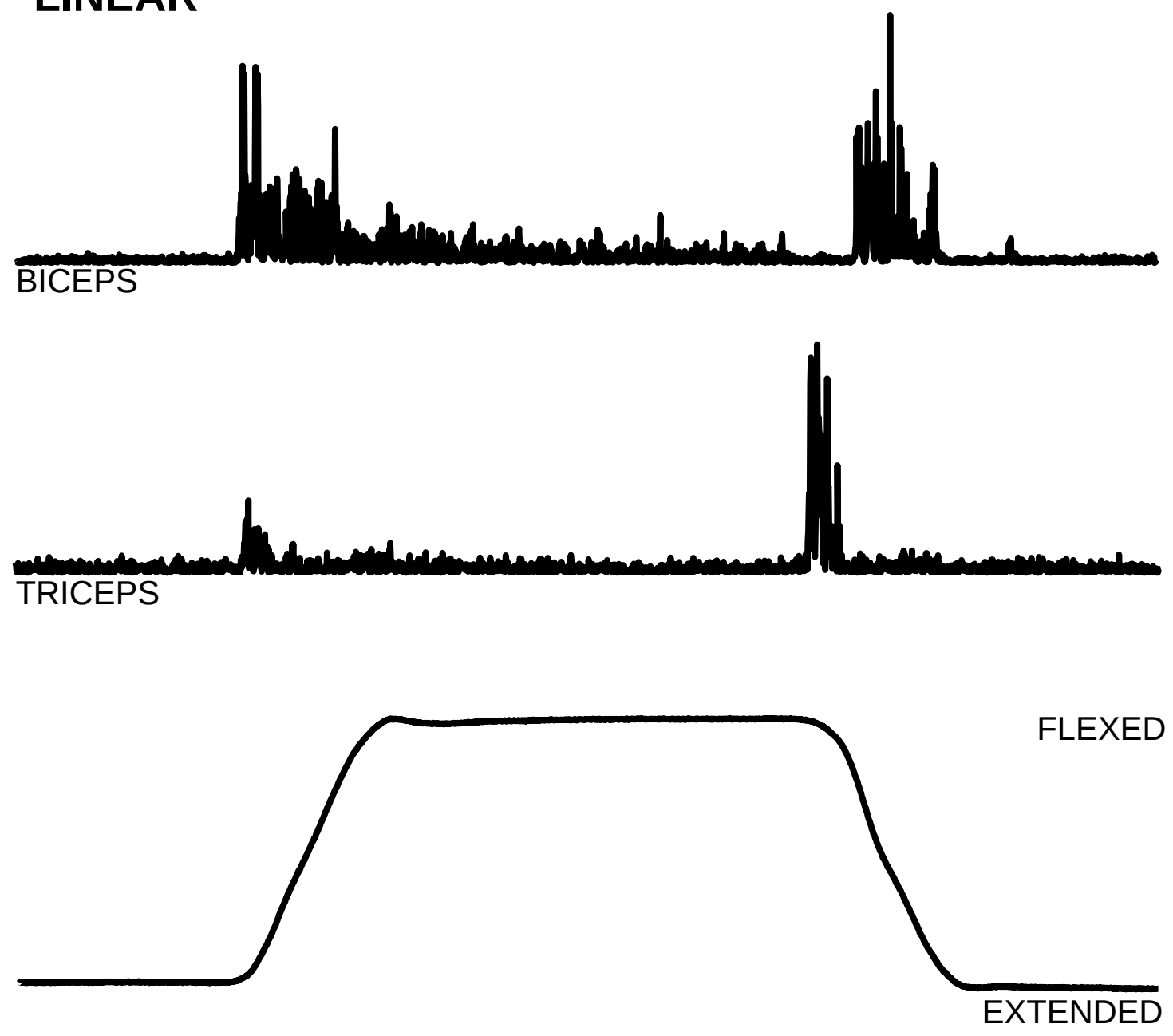
FIGURE 3

A

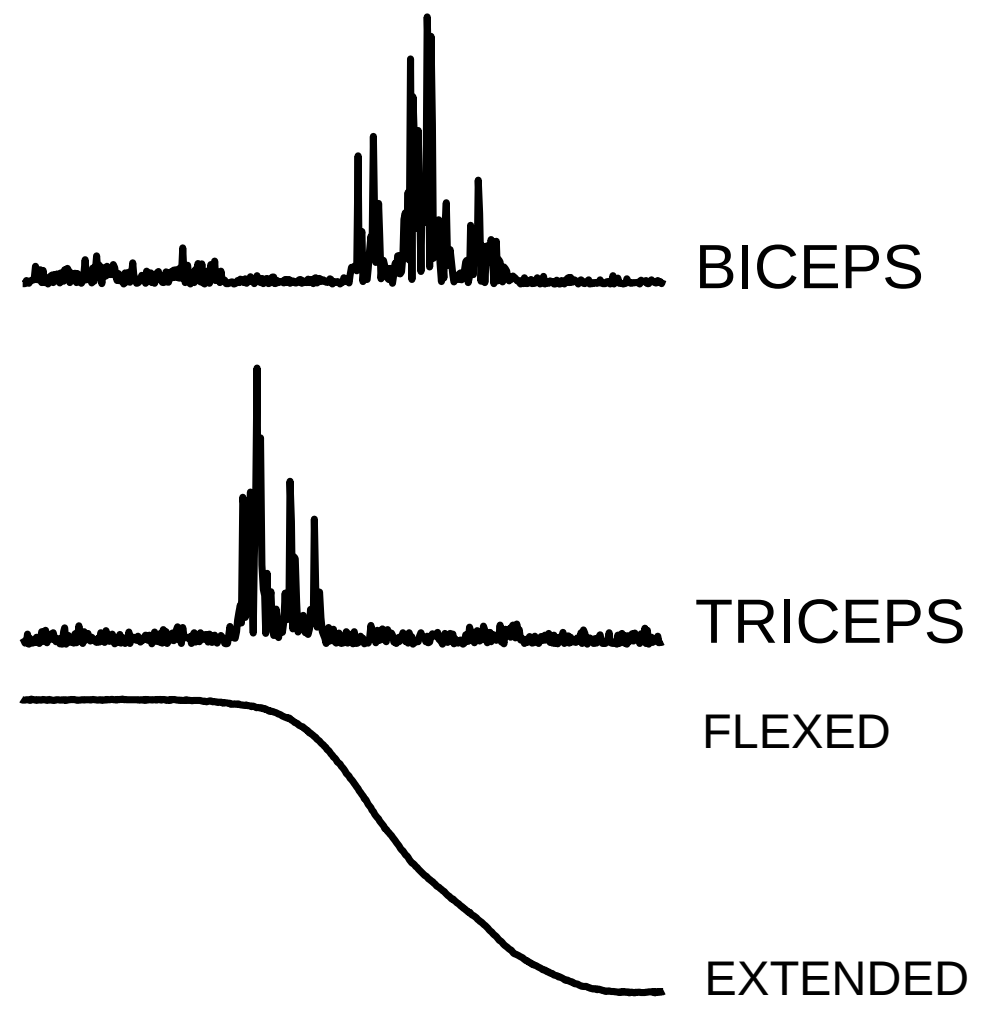

$200 \mathrm{~ms}$

BICEPS

EXTENDED

B
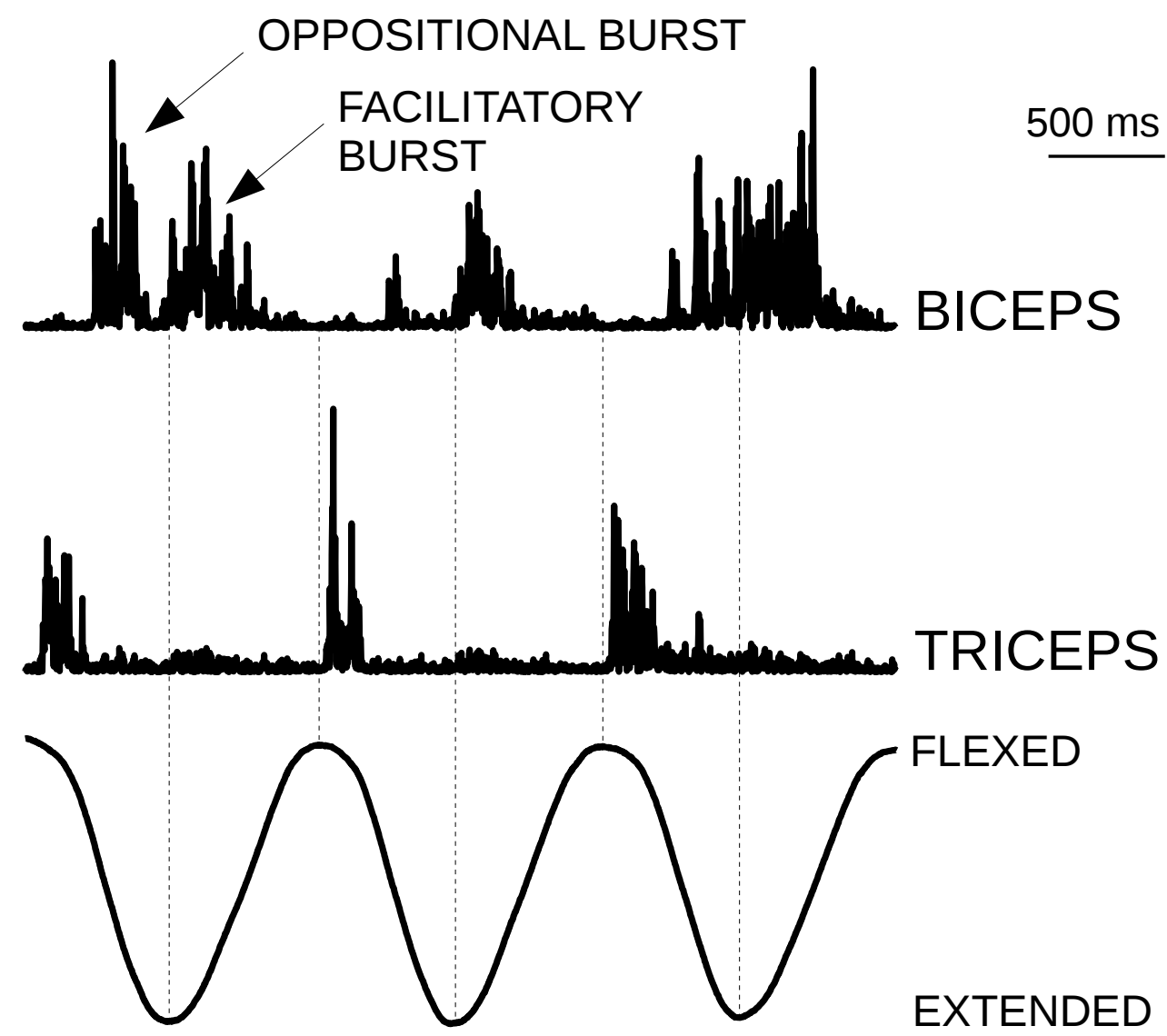
FIGURE 4

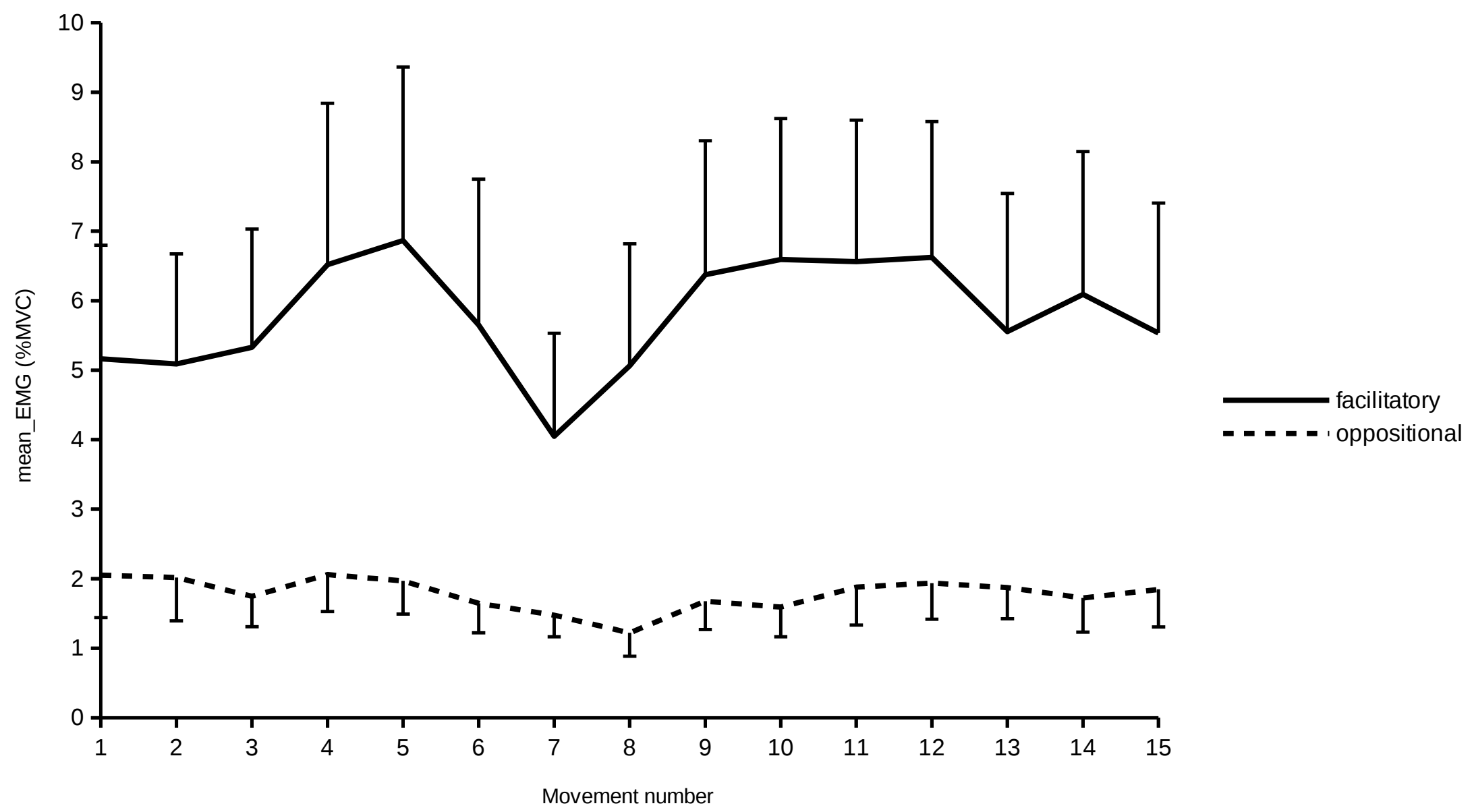


FIGURE 5

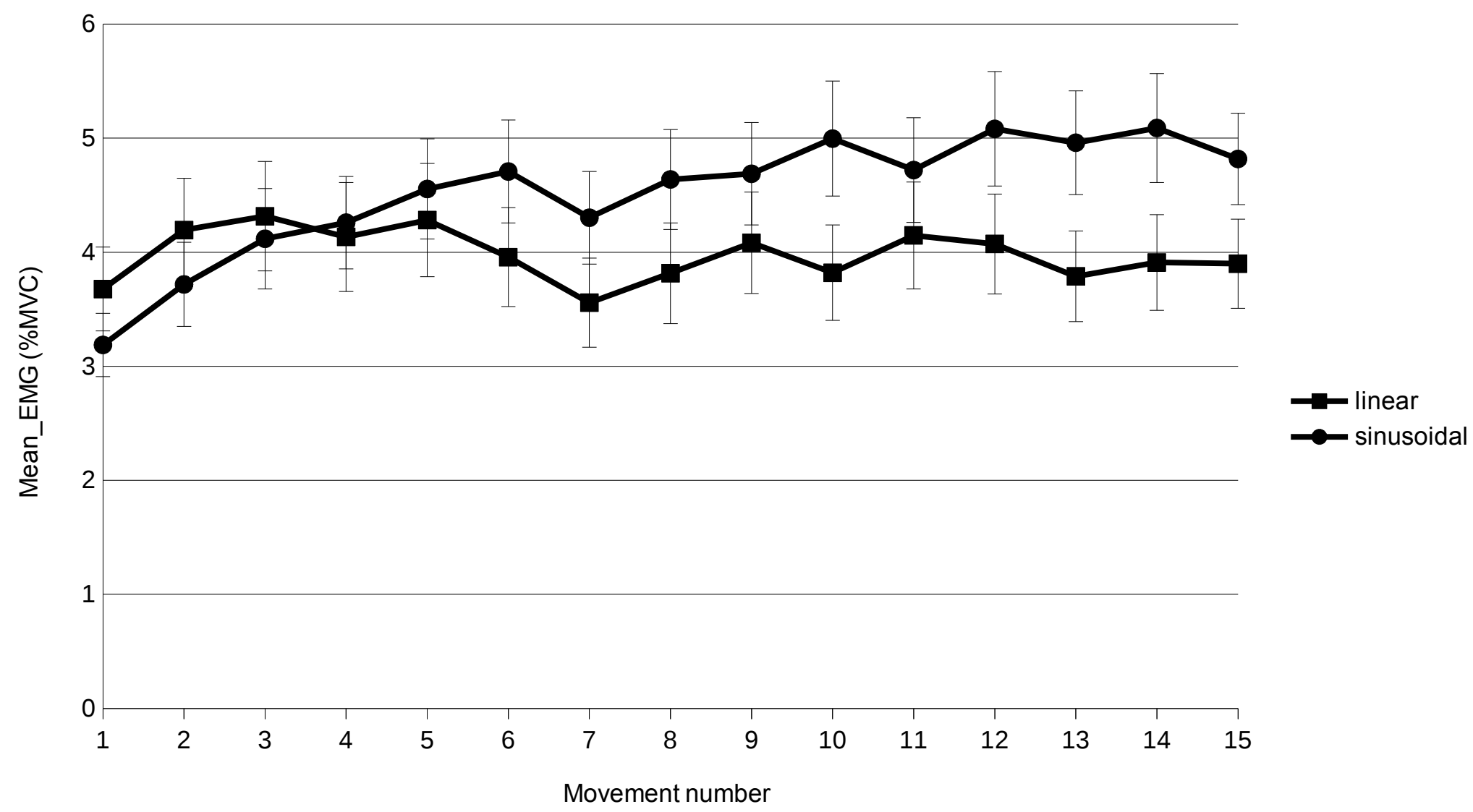

